ISSN 0103-9954

\title{
QUALIDADE DA SECAGEM CONVENCIONAL CONJUNTA DA MADEIRA DE CLONES DE TRÊS ESPÉCIES DE Eucalyptus sp.
}

\section{QUALITY OF GROUPED CONVENTIONAL KILN DRYING OF THREE SPECIES OF Eucalyptus sp. WOOD}

\author{
Djeison Cesar Batista $^{1}$ Ricardo Jorge Klitzke ${ }^{2}$ Márcio Pereira da Rocha ${ }^{3}$
}

\section{RESUMO}

A indústria brasileira comumente seca diferentes espécies de eucalipto em uma mesma carga de secagem convencional, seja pela dificuldade de identificação das espécies, híbridos e clones, seja para melhor utilizar o equipamento. No entanto, essa prática não é recomendada na literatura específica. O objetivo deste estudo foi verificar a qualidade da secagem convencional conjunta da madeira de Eucalyptus saligna, Eucalyptus grandis e Eucalyptus dunnii oriundas de plantios clonais. Para tal, foi realizada a secagem de três cargas com as três espécies conjuntamente em uma câmara-piloto de secagem convencional. Os critérios utilizados para analisar-se a qualidade da secagem foram: umidade final, rachaduras de topo, rachaduras de superfície, encanoamento, colapso, gradiente de umidade e tensões de secagem. Eucalyptus grandis apresentou a melhor qualidade de secagem, seguido pelo Eucalyptus saligna, e ambas as espécies possuem potencial para serem secas conjuntamente. Eucalyptus dunnii apresentou baixa qualidade de secagem, esta espécie não deve ser seca conjuntamente com Eucalyptus grandis e Eucalyptus saligna. A densidade básica e a retratibilidade foram bons critérios para a expectativa de qualidade da secagem de Eucalyptus spp., em que espécies menos densas e mais estáveis dimensionalmente apresentaram melhor qualidade de secagem. O programa de secagem utilizado deve ser modificado para a secagem futura dessas espécies, notadamente no tocante aos períodos de uniformização e condicionamento, que foram considerados insuficientes.

Palavras-chave: secagem artificial; eucalipto; defeitos de secagem.

\section{ABSTRACT}

The Brazilian industry commonly dries different species of eucalypt in a same load because of the difficulty of identifying the species, hybrids and clones, and also by optimizing the use of the kiln. However, this practice is not recommended in the literature. The aim of this work was to verify the quality of grouped conventional drying of Eucalyptus saligna, Eucalyptus grandis and Eucalyptus dunnii from clonal plantations. To this end, three loads were dried with the three species together in a pilot conventional dry kiln. The criteria used to analyze the quality of dried wood were final moisture content, end checks, cupping, collapse, moisture gradient and drying stresses. Eucalyptus grandis showed the best quality of drying, followed by Eucalyptus saligna, and both species have potential to be kiln dried together. Eucalyptus dunnii had the worst quality of drying, and should not be kiln dried together with Eucalyptus grandis and Eucalyptus saligna. The basic density and shrinkage were good parameters for the expected quality of the drying of eucalyptus species, in which less dense and more dimensionally stable species had

1 Engenheiro Florestal, Dr., Professor Adjunto do Departamento de Ciências Florestais e da Madeira, Universidade Federal do Espírito Santo, Av. Gov. Carlos Lindenberg, 316, Centro, CEP 29550-000, Jerônimo Monteiro (ES), Brasil.djeison.batista@ufes.br

2 Engenheiro Florestal, Dr., Professor Adjunto do Departamento de Engenharia e Tecnologia Florestal, Universidade Federal do Paraná, Av. Prefeito Lothário Meissner, 900, Jardim Botânico, CEP 80210-170, Curitiba (PR), Brasil. rklitzke@ufpr.br

3 Engenheiro Florestal, Dr., Professor Associado do Departamento de Engenharia e Tecnologia Florestal, Universidade Federal do Paraná, Av. Prefeito Lothário Meissner, 900, Jardim Botânico, CEP 80210-170, Curitiba (PR), Brasil.mprocha@ufpr.br

Recebido para publicação em 19/03/2012 e aceito em 25/10/2013 
better quality of drying. The drying schedule used must be modified to further drying of these species, especially regarding to uniformity and conditioning periods, which were considered insufficient.

Keywords: kiln drying; eucalypts; drying defects.

\section{INTRODUÇÃO}

Dentre as florestas plantadas que abastecem a indústria madeireira, o gênero Eucalyptus se tornou um dos mais importantes no Brasil, pela velocidade de desenvolvimento de suas árvores, facilidade de implantação em grandes maciços e versatilidade de aplicação de sua madeira.

A secagem é um processo obrigatório na geração de produtos de madeira sólida em função dos conhecidos benefícios que esta técnica agrega ao material, tanto na facilidade de trabalhabilidade em geral, quanto na redução do risco de ataque de agentes xilófagos.

Em secagem de madeiras existem princípios básicos que em uma mesma carga não devem ser secas espécies diferentes, nem tábuas de espessuras diferentes (GALVÃO e JANKOWSKY, 1985; HILDEBRAND, 1970; LANGRISH e WALKER, 2006; SIMPSON, 1991). Na prática, muitas vezes o volume das câmaras é superior ao volume serrado de uma determinada espécie, o que leva ao carregamento com espécies diferentes para, dentre outras causas, minimizar a ociosidade do equipamento e manter um determinado patamar produtivo. No caso de Eucalyptus spp., a dificuldade prática não é a falta de volume para carregar as câmaras, e sim, a identificação da madeira das diferentes espécies. Assim, em ambos os casos, não é raro diferentes espécies serem secas conjuntamente em uma mesma carga.

A densidade básica é a primeira propriedade a ser considerada como norteadora do agrupamento de diferentes espécies em uma mesma carga de secagem, porque esta se correlaciona diretamente com as demais propriedades físicas da madeira. Porém, o conhecimento de um maior número de características do comportamento de secagem das espécies levará a tomada de decisões mais acertadas.

O objetivo deste estudo foi verificar a qualidade da secagem convencional conjunta das madeiras de Eucalyptus saligna, Eucalyptus grandis e Eucalyptus dunnii oriundas de plantios clonais.

\section{MATERIAL E MÉTODOS}

Estudou-se as madeiras de Eucalyptus saligna, Eucalyptus grandis e Eucalyptus dunnii provenientes de plantios clonais homogêneos de 11 anos de idade, plantados em espaçamento 2,5 x 2,5 m, localizados em Telêmaco Borba, Paraná. De cada espécie foram utilizadas as primeiras toras (três metros) de 15 árvores com diâmetro a altura do peito (DAP) maior ou igual a $20 \mathrm{~cm}$, fuste retilíneo e aparentemente sadias, que foram colhidas no mesmo dia. Utilizaram-se tábuas tangenciais retiradas da porção intermediária do diâmetro, ou seja, entre a medula e a casca, de dimensões nominais iniciais de $30 \times 150 \times 3.000 \mathrm{~mm}$ (espessura, largura e comprimento).

De cada tábua original foram serradas três tábuas curtas de $745 \mathrm{~mm}$ de comprimento, que foram utilizadas na secagem convencional em câmara-piloto da marca Kiefer, com capacidade nominal de quatro metros cúbicos. A câmara possui sistema de aquecimento por serpentinas elétricas e sistema de umidificação abastecido por uma caldeira elétrica; o sistema de circulação de ar é composto por um ventilador com motor elétrico de $2 \mathrm{CV}$ e dois dampers, um de entrada e outro de saída de ar; a secagem é controlada por um sistema de gerenciamento, automatizado por um software específico, que permite monitorar, controlar e modificar as variáveis do processo por intermédio de um microcomputador. Entre cada tábua curta foi retirado um corpo de prova de $50 \mathrm{~mm}$ de comprimento que foi utilizado para a determinação da umidade inicial da carga, de acordo com o método gravimétrico.

Foram secas três cargas, em que cada uma correspondeu a uma pilha com dimensões aproximadas de $745 \mathrm{~mm}$ de largura, $500 \mathrm{~mm}$ de altura e $1.350 \mathrm{~mm}$ de comprimento, composta por 81 amostras (tábuas curtas), livres de defeitos (nós e medula, principalmente), 27 de cada espécie, resultando em nove camadas de madeira, cada camada com nove amostras.

As amostras foram empilhadas sobre um vagonete, com o comprimento disposto transversalmente ao fluxo de ar da câmara e separadas 
por tabiques de $25 \mathrm{~mm}$ de seção e $1.350 \mathrm{~mm}$ de comprimento. As amostras das diferentes espécies foram distribuídas homogeneamente, havendo tábuas de todas as espécies distribuídas pelas camadas da pilha. Para o controle da secagem foram utilizadas as amostras mais pesadas de cada lote, em que foram fixados dois pares de pinos sensores por amostra (um par de pinos curtos e outro de pinos longos). Utilizaram-se duas amostras de Eucalyptus dunnii, uma na parte anterior da primeira camada e outra na parte posterior da última camada; uma amostra de Eucalyptus saligna, na porção mediana da quarta camada; e outra de Eucalyptus grandis, na porção mediana da sexta camada. Foram utilizadas mais tábuas-controle de Eucalyptus dunnii porque se esperava que essa espécie fosse de mais difícil secagem do que as demais, por causa das maiores médias de densidade básica $\left(0,56 \mathrm{~g} / \mathrm{cm}^{3}\right)$ e contração volumétrica total $(18,71 \%)$, conforme Batista et al. (2010).

As três espécies foram secas conjuntamente, para que fossem submetidas às mesmas condições de secagem e, assim, permitisse uma avaliação igualitária da qualidade. Na Tabela 1 encontra-se o programa de secagem que foi utilizado nas três cargas, que foram secas até a umidade final de 10\%. A velocidade do ar utilizada foi $2,0 \mathrm{~m} / \mathrm{s}$, mantida constante ao longo de toda a secagem.

A avaliação da secagem foi feita de acordo com a ocorrência de defeitos, umidade final, gradiente de umidade e tensões de secagem. Estas avaliações foram feitas decorridas 24 horas do término da fase de resfriamento das cargas de secagem, conforme recomendado por Galvão e Jankowsky (1985).

Os defeitos avaliados foram: rachaduras de superfície, rachaduras de topo, encanoamento e colapso. Os defeitos foram classificados como presente ou ausente, utilizando-se $100 \%$ das amostras. Não se avaliaram os demais tipos de empenamentos por causa do comprimento reduzido das amostras.

Para a avaliação da umidade final, gradiente de umidade e tensões de secagem utilizou-se a metodologia proposta por Galvão e Jankowsky (1985), de acordo com a Figura 1, em que se utilizaram nove amostras/espécie/carga, correspondentes a $1 / 3$ de toda a madeira do estudo.

De cada amostra avaliada cortaram-se três corpos de prova (A, B e C) com $25 \mathrm{~mm}$ de

TABELA 1: Programa utilizado para a secagem das espécies Eucalyptus saligna, Eucalyptus grandis e Eucalyptus dunnii.

TABLE 1: Drying schedule for the species Eucalyptus saligna, Eucalyptus grandis and Eucalyptus dunnii.

\begin{tabular}{ccccccc}
\hline Umidade da Madeira $(\%)$ & $\mathrm{TBS}\left({ }^{\circ} \mathrm{C}\right)$ & $\mathrm{TBU}\left({ }^{\circ} \mathrm{C}\right)$ & $\mathrm{UR}(\%)$ & UE $(\%)$ & Tempo $(\mathrm{h})$ & PS \\
\hline Aquecimento & 40 & 39 & 94 & - & 3 & - \\
45 & 40 & 38 & 88 & 18 & - & 2,5 \\
35 & 44 & 41 & 84 & 16 & - & 2,2 \\
31 & 46 & 42 & 78 & 14 & - & 2,2 \\
28 & 50 & 45 & 77 & 14 & - & 2,0 \\
25 & 54 & 48 & 71 & 12 & - & 2,1 \\
20 & 58 & 49 & 64 & 10 & - & 2,0 \\
17 & 60 & 49 & 55 & 8 & - & 2,1 \\
15 & 62 & 49 & 48 & 7 & - & 2,1 \\
12 & 66 & 51 & 46 & 6 & - & 2,0 \\
10 & 66 & 47 & 35 & 5 & - & 2,0 \\
Uniformização-10 & 64 & 56 & 66 & 10 & 8 & - \\
Condicionamento-10 & 62 & 56 & 73 & 12 & 8 & - \\
Resfriamento-10 & 44 & 35 & 55 & 9 & 4 & - \\
\hline
\end{tabular}

Em que: TBS $=$ temperatura de bulbo seco; $\mathrm{TBU}=$ temperatura de bulbo úmido; $\mathrm{UR}=$ umidade relativa; $\mathrm{UE}=$ umidade de equilíbrio; PS = potencial de secagem. 


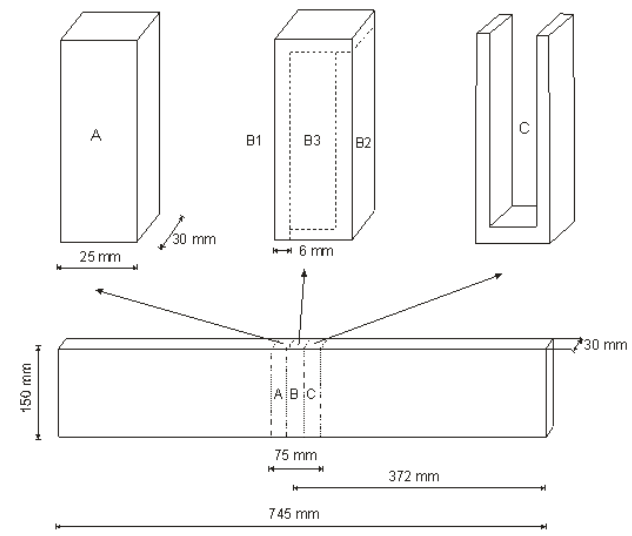

FIGURA 1: Corpos de prova utilizados na avaliação da umidade final, gradiente de umidade e tensões de secagem (adaptado de GALVÃO e JANKOWSKY, 1985). Sem escala.

FIGURE 1: Specimens utilized in the evaluation of final moisture content, moisture gradient and drying stresses test (adapted from GALVÃO and JANKOWSKY, 1985). Not to scale.

comprimento cada. O corpo de prova A foi utilizado para a determinação da umidade final, de acordo com o método gravimétrico. O corpo de prova $\mathrm{B}$ foi utilizado para a avaliação do gradiente de umidade e, para isto, foi serrado dando origem a outros três corpos de prova (B1, B2 e B3). B1 e B2 foram utilizados para a determinação da umidade da superfície das amostras, enquanto B3 foi utilizado para a determinação da umidade no centro das mesmas, de acordo com o método gravimétrico. Calcularam-se dois gradientes de umidade: entre os corpos de prova B3 e B1 e entre B3 e B2, conforme as Equações 1 e 2.

$$
\begin{aligned}
& \mathrm{G} 1=\mathrm{UB} 3-\mathrm{UB} 1 \\
& \mathrm{G} 2=\mathrm{UB} 3-\mathrm{UB} 2
\end{aligned}
$$

Em que - G1: gradiente de umidade 1 (\%); G2: gradiente de umidade $2(\%)$; UB1: umidade na superfície 1 da amostra (\%); UB2: umidade na superfície 2 da amostra (\%); UB3: umidade no interior da amostra (\%).

O corpo de prova $\mathrm{C}$ foi utilizado para a avaliação da presença de tensões residuais de secagem, de acordo com o "teste do garfo". Cada corpo de prova foi classificado como: tensionado, livre de tensões ou com tensões invertidas, conforme Galvão e Jankowsky (1985).

$\mathrm{Na}$ Tabela 2 encontram-se os critérios utilizados para comparar a secagem realizada neste trabalho quanto à duração do tempo de secagem e incidência de defeitos.

Foi realizada análise estatística somente para os resultados, por espécie, de umidade inicial, umidade final e gradiente de umidade, que foram avaliados de acordo com a ANOVA a 5\% de significância. As análises foram feitas comparando-se as espécies entre si, e nas situações em que a hipótese da nulidade (H0) foi rejeitada, aplicou-se o teste de Tukey (5\% de significância) para diferenciação das médias. A análise dos gradientes de umidade dentro de uma mesma

TABELA 2: Critérios utilizados para a classificação da duração da secagem e incidência de defeitos de secagem.

TABLE 2: Criteria used in the evaluation of drying time and incidence of drying defects.

\begin{tabular}{cccc}
\hline${\text { Tempo de secagem }(\mathrm{h})^{1}}^{1}$ & Classificação $^{1}$ & Amostras que apresentaram defeitos (\%) $^{2}$ & Classificação $^{2}$ \\
\hline Menos de 96 & Muito rápida & $0-10$ & Ausente \\
$96-192$ & Rápida & $11-30$ & Pequena \\
192,1-288 & $\begin{array}{c}\text { Moderadamente } \\
\text { rápida } \\
\text { Moderadamente } \\
\text { lenta }\end{array}$ & $31-50$ & Moderada \\
384,1-384 & Lenta & $51-100$ & Grande \\
Mais de 480 & Muito lenta & - & - \\
\hline Fonte: ${ }^{1}$ Brasil (1981) $\mathrm{e}^{2}$ Martins (1984) citados por Brandão (1989). & - \\
\hline
\end{tabular}


espécie foi realizada de acordo com o teste t a $5 \%$ de significância. A análise estatística foi realizada no software Microsoft Excel ${ }^{\circledR}$ versão 2010.

\section{RESULTADOS E DISCUSSÃO}

Na Tabela 3 encontram-se os resultados referentes às três cargas de secagem. As umidades médias iniciais e finais correspondem à média dos oito pares de pinos sensores, fornecidas pelo software de controle de secagem. A umidade inicial média entre as cargas foi heterogênea, apresentando uma diferença máxima de 21 pontos percentuais. Esta diferença influenciou no tempo de secagem, em que maiores umidades iniciais corresponderam a ciclos de secagem mais longos.

A duração média da secagem convencional em câmara piloto foi de 453,3 horas, correspondente a 18,9 dias. De acordo com Brasil (1981) citado por Brandão (1989), a duração da secagem foi classificada como lenta, o que já era esperado dado ao conhecimento da dificuldade de secagem da madeira de Eucalyptus sp., por causa da sua baixa permeabilidade.

No trabalho de Ciniglio (2000), a secagem de tábuas de $25 \mathrm{~mm}$ de Eucalyptus grandis e Eucalyptus urophylla levou 25 e 22 dias, respectivamente. Jankowsky et al. (2000) citado por Jankowsky et al. (2003) secaram madeira de Eucalyptus grandis de $40 \mathrm{~mm}$ por 77 dias ao ar, seguidos de mais 15 dias de secagem convencional. Os resultados obtidos, aliados aos de Ciniglio (2000), reforçam a dificuldade da secagem da madeira de Eucalyptus spp., por causa da sua baixa permeabilidade. ser desenvolvidos de tal forma a induzirem a uma secagem lenta. Ainda, a realização de pré-secagem pode ser utilizada como uma alternativa viável para se reduzir o tempo de secagem em câmara da madeira de eucalipto.

A umidade final média foi igual para todas as cargas por causa do controle da secagem realizado pelo software específico. Assim, na última etapa do programa (resfriamento), quando a média dos pinos sensores atingia $10 \%$, a câmara era desligada automaticamente. No entanto, conforme será observado adiante, esse resultado não correspondeu à realidade, em função da heterogeneidade $\mathrm{e}$ dificuldade da secagem da madeira de eucalipto e, também, pela precisão dos pinos sensores, que é de um ponto percentual.

Apesar de não ter sido feita uma análise específica, com base na umidade registrada pelos pinos sensores, notou-se que a secagem da madeira de Eucalyptus dunnii foi mais lenta, conforme esperado, por sua maior densidade básica (BATISTA et al., 2010). Como a troca de fases do programa de secagem foi realizada pelo sistema de automação da câmara, em que o software comanda essa troca pela média de umidade dos pinos sensores, pode-se atribuir que toda a secagem foi controlada com base na madeira de Eucalyptus dunnii. Isso indica que, para o mesmo programa de secagem, secando-se apenas madeira de Eucalyptus grandis e Eucalyptus saligna, ou ambas conjuntamente, o tempo de secagem médio será menor do que a secagem das três espécies conjuntamente.

Observou-se pequena variação entre a taxa de secagem média, indicando um comportamento similar da secagem entre as cargas. A carga 3, de maior umidade inicial média, apresentou a maior taxa média influenciada provavelmente pela retirada de mais massa de água na fase capilar. A taxa de secagem média de $0,141 \% / \mathrm{h}$ foi similar aos 0,150 $\% / \mathrm{h}$ obtido por Santos et al. (2003) para a madeira de cerne de Eucalyptus grandis, aos 16 anos, com $20 \mathrm{~mm}$ de espessura, reafirmando a dificuldade de secagem da madeira de eucalipto.

As médias de umidade inicial e final da madeira das diferentes espécies encontram-se apresentadas na Tabela 4.

A homogeneidade da umidade inicial das cargas observada entre as espécies foi um resultado

TABELA 3: Resultados das cargas de secagem.

TABLE 3: Kiln run results.

\begin{tabular}{ccccc}
\hline Carga & Umidade inicial média (\%) & Duração $(\mathrm{h})$ & Umidade final média (\%) & Taxa de secagem média (\%/h) \\
\hline 1 & 65 & 429 & 10 & 0,128 \\
2 & 71 & 446 & 10 & 0,137 \\
3 & 86 & 485 & 10 & 0,157 \\
\hline Média & 74 & 453,3 & 10 & 0,141 \\
\hline
\end{tabular}


desejável, indicando que todas elas foram secas de uma condição igual, visto que tábuas mais úmidas de madeiras refratárias requerem, de maneira geral, maior tempo de secagem. Assim, a taxa de secagem em câmara convencional foi regulada pelos fatores intrínsecos a cada espécie, embora as médias de umidade inicial não tenham diferido, bem como as condições de secagem referentes ao equipamento utilizado foram as mesmas. No entanto, analisandose o coeficiente de variação, observou-se elevada heterogeneidade da umidade das peças dentro de uma mesma espécie, resultando em heterogeneidade da carga, o que não dificultou a homogeneidade da umidade final.

A espécie Eucalyptus saligna apresentou a maior umidade inicial média, seguida por Eucalyptus grandis e Eucalyptus dunnii, porém, não diferiram estatisticamente. Esperava-se que o Eucalyptus dunnii apresentasse a menor umidade inicial em razão da sua maior densidade básica, o que não ocorreu na prática. Os resultados de umidade inicial por espécie foram inferiores aos obtidos por outros autores (CINIGLIO, 2000; SEVERO, 2000; ROCHA, 2000), porém, não interferiram na condução do experimento por ainda possuírem suficiente quantidade de água capilar, classificando a madeira como em estado saturado.

O Eucalyptus grandis foi a única espécie com umidade final média abaixo dos $10 \%$ propostos pelo programa de secagem, porém não diferiu do Eucalyptus saligna. Conforme Batista et al. (2010), a densidade básica média da madeira dessas espécies foi de $0,45 \mathrm{~g} / \mathrm{cm}^{3}$ e $0,46 \mathrm{~g} / \mathrm{cm}^{3}$, respectivamente, inferiores aos $0,56 \mathrm{~g} / \mathrm{cm}^{3}$ do Eucalyptus dunnii. Assim, os resultados das propriedades físicas já apontavam para a maior facilidade de secagem dessas duas espécies.
Ciniglio (2000) obteve para as espécies Eucalyptus grandis e Eucalyptus urophylla umidade final média 2 pontos percentuais superiores ao estipulado pelo programa de secagem, considerando este valor elevado, causado provavelmente por um período de uniformização insuficiente. O Eucalyptus dunnii apresentou umidade final média 1,45 ponto percentual superior aos $10 \%$ propostos pelo programa de secagem, corroborando com a expectativa de maior dificuldade de secagem desta espécie, associada à maior densidade básica. Mesmo sendo a espécie de secagem mais difícil, Eucalyptus dunnii apresentou umidade final média mais próxima do desejado do que as espécies estudadas por Ciniglio (2000).

A umidade final entre as peças foi mais homogênea do que a umidade inicial, representada pelos menores coeficientes de variação, indicando que a secagem convencional é uma técnica benéfica em relação a esse aspecto. Notou-se ainda que a diferença de umidade entre as amostras de Eucalyptus dunnii foi a maior, expressa pelo maior coeficiente de variação $(17,07 \%)$. O período de uniformização utilizado (oito horas) foi considerado insuficiente, devendo ser aumentado, principalmente para posteriores secagens de Eucalyptus dunnii.

Esses resultados confirmam a importância do conhecimento das propriedades físicas das madeiras que se deseja secar, principalmente espécies de comportamento de secagem pouco conhecido.

Na Tabela 5 encontram-se os resultados da porcentagem das tábuas com defeitos de secagem por espécie. A classificação entre parênteses referese ao critério de tendência de incidência de defeitos de secagem (Tabela 2). De cada espécie foram avaliadas 81 amostras. Nota-se que a soma das

TABELA 4: Umidades médias iniciais e finais da madeira de Eucalyptus saligna, Eucalyptus grandis e Eucalyptus dunnii.

TABLE 4: Means of initial and final wood moisture content of Eucalyptus saligna, Eucalyptus grandis and Eucalyptus dunnii.

\begin{tabular}{ccccc}
\hline \multirow{2}{*}{ Espécie } & \multicolumn{4}{c}{$(\%)$} \\
\cline { 2 - 5 } & Umidade inicial & Coeficiente de variação & Umidade final & Coeficiente de variação \\
\hline Eucalyptus saligna & $79,40 \mathrm{a}$ & 24,98 & $10,08 \mathrm{a}$ & 9,59 \\
Eucalyptus grandis & $77,36 \mathrm{a}$ & 25,64 & $9,96 \mathrm{a}$ & 10,84 \\
Eucalyptus dunnii & $76,45 \mathrm{a}$ & 21,30 & $11,45 \mathrm{~b}$ & 17,07 \\
\hline Média & 77,78 & 23,95 & 10,52 & 14,90 \\
\hline
\end{tabular}

Em que: Médias seguidas de uma mesma letra, em uma mesma coluna, não diferem entre si de acordo com o teste de

Tukey a 5\% de significância. 
porcentagens de defeitos ultrapassa $100 \%$, porque uma mesma tábua pode apresentar mais de um tipo de defeito.

O Eucalyptus saligna apresentou a menor porcentagem de amostras com rachaduras de topo, seguido por Eucalyptus grandis e Eucalyptus dunnii, no entanto, a incidência deste defeito para todas as espécies foi classificada como "ausente". Outros autores que também avaliaram madeira de eucalipto obtiveram a mesma classificação para a incidência de rachaduras de topo, tal como Ciniglio (1998) para as espécies Eucalyptus grandis e Eucalyptus urophylla. Mellado (1993), por exemplo, analisou tábuas de $30 \mathrm{~mm}$ de espessura de Eucalyptus grandis aos 14 anos e relatou 5,6\% das amostras com este defeito, enquanto Severo (2000) obteve, para o programa de secagem mais brando avaliado, $10 \%$ de tábuas de Eucalyptus dunnii com rachaduras de topo.

Da mesma forma que as rachaduras de topo, todas as espécies receberam a classificação "ausente" quanto à incidência de rachaduras de superfície. Destaca-se o Eucalyptus grandis, no qual nenhuma das amostras apresentou este defeito, enquanto o Eucalyptus dunnii teve a maior porcentagem, comportamento igual ao das rachaduras de topo.

Quanto ao encanoamento, as espécies foram classificadas com "grande" incidência deste defeito em que a porcentagem de tábuas encanoadas foi maior do que aquelas obtidas por outros autores (MELLADO, 1993; CINIGLIO, 2000; SEVERO, 1998). De acordo com Batista et al. (2010), a madeira das espécies estudadas é instável dimensionalmente, em especial por ser composta, majoritariamente, por lenho juvenil (11 anos), em que a contração volumétrica total média e o coeficiente de anisotropia médio foram de 15,16\%/2,06, 14,10\%/2,05 e $18,71 \% / 2,36$, respectivamente para Eucalyptus saligna, Eucalyptus grandis e Eucalyptus dunnii.
Portanto, tal incidência de encanoamento em tábuas tangenciais deveu-se à instabilidade dimensional da madeira.

Analisando-se o colapso, as espécies receberam classificações diferentes entre si, de acordo com o critério adotado. Os $55,56 \%$ das tábuas com colapso do Eucalyptus dunnii foram superiores aos $35 \%$ obtidos no programa de secagem mais suave testado por Severo (2000), porém, inferiores aos $67,5 \%$ e $87,5 \%$ obtidos nos outros dois programas (mais acelerados). Destaca-se que o autor utilizou árvores de 16 anos, tábuas tangenciais de $24 \mathrm{~mm}$, e os programas de secagem empregados foram desenvolvidos especificamente com etapas de recuperação de colapso da madeira. Tais resultados corroboram com a classificação "grande" de incidência de colapso nesta espécie, sugerindo para programas posteriores à realização da etapa de secagem inicial (remoção da água capilar) mais suave. Os $35,80 \%$ de tábuas com colapso do Eucalyptus grandis foram superiores aos $8,3 \%$ obtidos por Ciniglio (2000) para a mesma espécie aos 18 anos em tábuas de 25 mm de espessura, corroborando com a classificação "moderada" de incidência deste tipo de defeito nesta espécie. O Eucalyptus saligna foi a única espécie que recebeu a classificação "pequena" de incidência de colapso, de acordo com os critérios adotados. Porém, a utilização de fases iniciais de secagem mais suaves e técnicas de recuperação de colapso (MELLADO, 1993; SEVERO, 2000), nas aplicações que necessitam de madeira de melhor qualidade, podem ser viáveis para a redução da porcentagem de colapso em todas as espécies.

$\mathrm{Na}$ Tabela 6 encontram-se as médias por espécie do gradiente de umidade e o tipo de tensão de secagem, fornecido pelo teste do garfo. Para tais avaliações foram utilizadas 81 amostras por espécie. Os gradientes de umidade 1 e 2 equivalem

TABELA 5: Porcentagem de tábuas de Eucalyptus saligna, Eucalyptus grandis e Eucalyptus dunnii com defeitos de secagem.

TABLE 5: Percentage of Eucalyptus saligna, Eucalyptus grandis and Eucalyptus dunnii boards with drying defects.

\begin{tabular}{ccccc}
\hline \multirow{2}{*}{ Espécie } & \multicolumn{4}{c}{$\%$} \\
\cline { 2 - 5 } & Rachaduras de topo & Rachaduras de superfície & Encanoamento & Colapso \\
\hline Eucalyptus saligna & 4,94 (ausente) & 3,70 (ausente) & 67,90 (grande) & 28,40 (pequena) \\
Eucalyptus grandis & 7,91 (ausente) & 0 (ausente) & 60,49 (grande) & 35,80 (moderada) \\
Eucalyptus dunnii & 9,88 (ausente) & 4,94 (ausente) & 74,07 (grande) & 55,56 (grande) \\
\hline
\end{tabular}


à dedução da umidade final do centro em relação às superfícies das tábuas.

Como não foi observado o tensionamento de secagem do tipo invertido, decidiu-se reclassificar as tábuas tensionadas em tensionamento suave e forte. O tensionamento suave foi aquele nos quais os extremos dos garfos não se tocavam, em contraposição ao tensionamento forte, em que estes se tocavam.

$\mathrm{O}$ gradiente de umidade 2 referiu-se à face superior das tábuas, e notou-se que este gradiente foi inferior ao gradiente 1 (face inferior), em termos absolutos. Contudo, não houve diferença significativa entre os gradientes das duas faces para uma mesma espécie.

O Eucalyptus grandis apresentou os menores gradientes médios de umidade, não diferindo estatisticamente do Eucalyptus saligna, enquanto o Eucalyptus dunnii apresentou os maiores gradientes médios de umidade. Os gradientes de umidade do Eucalyptus saligna e Eucalyptus grandis foram semelhantes aos 0,6 pontos percentuais obtidos por Mellado (1993) para a madeira de Eucalyptus grandis. Ainda de acordo com o autor, mesmo gradientes de umidades pequenos levam à incidência de tensões de secagem nas tábuas. O gradiente de umidade de 2,37\% apresentado pelo Eucalyptus dunnii foi inferior aos 4 pontos percentuais obtidos por Ciniglio (1998) para a madeira de Eucalyptus grandis, porém, superior aos 2 pontos percentuais obtidos pela mesma autora para Eucalyptus urophylla.

O Eucalyptus grandis não apresentou nenhum corpo de prova com tensionamento forte $\mathrm{e}$ foi a espécie com a maior porcentagem de amostras livres de tensões de secagem, com 18,52\%; enquanto o Eucalyptus dunnii foi aquela na qual todos os corpos de prova apresentaram tensões de secagem, com a maior porcentagem de amostras com tensionamento forte, $59,26 \%$, e a menor porcentagem de amostras com tensionamento suave, $40,74 \%$.

Assim, o gradiente de umidade foi diretamente relacionado com a presença de tensões de secagem, no qual quanto maior o gradiente, maior a incidência de tensões de secagem. Estes resultados estão de acordo com aqueles de umidade final (Tabela 5) e das propriedades físicas (BATISTA et al., 2010), nos quais o Eucalyptus dunnii foi a espécie de secagem mais difícil, em oposição a Eucalyptus grandis e Eucalyptus saligna.

A grande porcentagem de amostras com tensões de secagem sugere que as suas superfícies estavam sob compressão, enquanto o centro estava sob tração (SKAAR, 1972). Este resultado reforça que os tempos de uniformização e condicionamento utilizados (ambos de oito horas) foram insuficientes, indicando que, para futuras secagens dessas espécies, os mesmos devam ser aumentados, principalmente para o Eucalyptus dunnii.

O gradiente de umidade e as tensões de secagem por espécie também se correlacionaram diretamente com os defeitos de secagem, no qual quanto maior o gradiente de umidade, mais tensionadas foram as amostras, levando a um maior índice de defeitos.

\section{CONCLUSÕES}

De acordo com o programa de secagem e as características da madeira das espécies utilizadas, pode-se concluir para a secagem convencional conjunta:

- O Eucalyptus grandis apresentou a

TABELA 6: Gradiente de umidade e tensões de secagem da madeira de Eucalyptus saligna, Eucalyptus grandis e Eucalyptus dunnii.

TABLE 6: Moisture gradient and drying stresses of Eucalyptus saligna, Eucalyptus grandis and Eucalyptus dunnii wood.

\begin{tabular}{lccccc}
\hline \multirow{2}{*}{ Espécie } & \multicolumn{2}{c}{ Gradiente de umidade (pontos percentuais) } & \multicolumn{3}{c}{ Tensões de secagem (\%) } \\
\cline { 2 - 6 } & 1 & 2 & Livre & Suave & Forte \\
\hline Eucalyptus saligna & $0,62 \mathrm{aA}$ & $0,50 \mathrm{aA}$ & 7,41 & 88,89 & 3,70 \\
Eucalyptus grandis & $0,58 \mathrm{aA}$ & $0,49 \mathrm{aA}$ & 18,52 & 81,48 & 0 \\
Eucalyptus dunnii & $2,37 \mathrm{bA}$ & $2,10 \mathrm{bA}$ & 0 & 40,74 & 59,26 \\
\hline
\end{tabular}

Em que: Médias seguidas de uma mesma letra minúscula, em uma mesma coluna, não diferem entre si de acordo com o teste de Tukey a 5\% de significância. Médias seguidas de uma mesma letra maiúscula, em uma mesma linha, não diferem entre si de acordo com o teste t a 5\% de significância. 
melhor qualidade de secagem, seguido por Eucalyptus saligna, e ambas as espécies possuem potencial para serem secas conjuntamente.

- O Eucalyptus dunnii apresentou baixa qualidade de secagem, e esta espécie não deve ser seca conjuntamente com Eucalyptus grandis e Eucalyptus saligna.

- Adensidade básica e a retratibilidade foram bons parâmetros para a expectativa de qualidade da secagem de Eucalyptus, em que espécies menos densas e mais estáveis dimensionalmente apresentaram melhor qualidade de secagem.

- O programa de secagem utilizado deve ser modificado para secagens futuras dessas espécies, especialmente no tocante aos períodos de uniformização e condicionamento, que foram considerados insuficientes.

\section{AGRADECIMENTOS}

Os autores expressam seus agradecimentos ao $\mathrm{CNPq}$ (Conselho Nacional de Desenvolvimento Científico e Tecnológico) pela concessão da bolsa de mestrado ao primeiro autor.

\section{REFERÊNCIAS BIBLIOGRÁFICAS}

BATISTA, D. C.; KLITZKE, R. J.; SANTOS, C. V. T. Densidade básica e retratibilidade da madeira de clones de três espécies de Eucalyptus. Ciência Florestal, Santa Maria, v. 20, n. 4, p. 665-674, 2010. BRANDÃO, A. T. O. Determinação de Metodologia para a Indicação de Programas de Secagem de Madeiras. Piracicaba, 1989. 100 f. Dissertação (Mestrado em Ciências Florestais) - Escola Superior de Agricultura Luiz de Queiroz, Universidade de São Paulo, 1989.

CINIGLIO, G. Avaliação da secagem de madeira serrada de Eucalyptus grandis e Eucalyptus urophylla. 1998. 73 f. Dissertação (Mestrado em Ciências e Tecnologia de Madeiras) - Escola Superior de Agricultura Luiz de Queiroz, Universidade de São Paulo, 1998.

GALVÃO, A. P. M.; JANKOWSKY, I. P. Secagem racional da madeira. São Paulo: Nobel, 1985. $111 \mathrm{p}$.

HILDEBRAND, R. Kiln drying of sawn timber. Plochingen: Richard Schorndorfer, 1970. 199 p.

JANKOWSKY, I. P; SANTOS, G. R. V.; ANDRADE, A. Secagem da madeira serrada de eucalipto. Circular Técnica do IPEF, Piracicaba, n. 199, 2003.

LANGRISH, T.; WALKER, J. Drying of timber. In. WALKER, J. C.F. Primary wood processing: principles and practice. 2a edição. Dordrecht: Springer, 2006. Capítulo 8, p. 251-295.

MELLADO, E. C. R. Contribuição ao desenvolvimento tecnológico para a utilização de madeira serrada de Eucalyptus grandis (Hill ex Maiden) na geração de produtos com maior valor agregado. 1993. 133 f. Dissertação (Mestrado em Ciências Florestais) - Universidade Federal do Paraná, Curitiba, 1993.

ROCHA, M. P. Eucalyptus grandis Hill ex Maiden e Eucalyptus dunnii Maiden como fontes de matéria-prima para Serrarias. 2000.185 f. Tese (Doutorado em Ciências Florestais) -Universidade Federal do Paraná, Curitiba, 2000.

SANTOS, G. R. V.; JANKOWSKY, I. P.; ANDRADE, A. Curva característica de secagem para madeira de Eucalyptus grandis. Scientia Forestalis, Piracicaba, n. 63, p. 214-220, 2003.

SEVERO, E.T.D. Qualidade da secagem de madeira serrada de Eucalyptus dunnii. Ciência Florestal, Santa Maria, v.10, n.1, p.109-124, 2000.

SIMPSON, W. T. Dry kiln operator's manual. Madison: United States Department of Agriculture, Forest Service, Forest Products Laboratory, 1991. $274 \mathrm{p}$.

SKAAR, C. Water in Wood. Syracuse: Syracuse University Press, 1972. 218 p. 